\title{
Welcome to the year 2022
}

\author{
Nadia Magnenat-Thalmann ${ }^{1}$
}

Published online: 22 January 2022

(c) The Author(s), under exclusive licence to Springer-Verlag GmbH Germany, part of Springer Nature 2022

We are very happy to announce that the Visual Computer Journal has progressed significantly during the year 2021 although we had a hard time due to the Covid-19. We are so glad that the activity of the journal has continued as usual even with the pandemic going on.

During 2021, the submissions rate has strongly increased, and we have improved our Impact Factor to 2.601. Although we try to keep an acceptance rate of $20 \%$, we have to desk reject quite a lot of papers. The reason is that many papers purely in vision are wrongly submitted to the Visual Computer, which is mainly a computer graphics journal. Our journal accepts papers in vision or image processing if they are related to computer graphics.

We take this opportunity to thank our Associate Editors and Reviewers for their tremendous work in reviewing and handling each paper carefully.

Throughout 2021, some Associate Editors have left the editorial board of the Visual Computer. We would like to thank them for their great work and contribution. They are:

- Neeharika Adabala, India

- Florent Lafarge, France

- Bruno Levy, INRIA, France

- Liang Lin, Sun Yat-sen University, China

- Masayuki Nakajima, Japan

- Bodo Rosenhahn, Leibniz University of Hannover, Germany

- Jianbin Shen, China

- Qixiang Ye, Chinese Academy of Sciences, China

The editorial board of the Visual Computer is renewed regularly. This year, due to the high increase of submissions of papers, we have invited several experts in different fields to join the editorial board. We welcome our new Associate Editors who are listed below:

Nadia Magnenat-Thalmann

thalmann@miralab.ch

1 MIRALab-CUI, University of Geneva, Battelle, Building A, 7, Route de Drize, 1227 Carouge, GE, Switzerland
- Antonio Agudo, CSIC-UPC, Spain

- Imon Banerjee, Emory University School of Medicine, USA

- Sebastiano Battiato, Università di Catania, Italy

- Tolga Birdal, Stanford University, USA

- Ladislau Boloni, University of Central Florida, USA

- Zhonggui Chen, Xiamen University, China

- Sunghyun Cho, POSTEC, South Korea

- Jan Egger, Graz University of Technology, Austria

- Antonino Furnari, University of Catania, Italy

- Zhenhua Guo, Alibaba Group, China

- Dakai Jin, PAII Inc., USA

- Stefano Mattoccia, University of Bologna, Italy

- Tae-Hyun Oh, POSTECH, South Korea

- Jinshan Pan, Nanjing University of Science and Technology, China

- Andrea Prati, Università degli Studi di Parma, Italy

- Jonathan Roberts, Bangor University, UK

- Thomas Schultz, University of Bonn, Germany

- Bernie Tiddeman, Aberystwyth University, UK

- Zhigang Tu, Wuhan University, China

- Nannan Wang, Xidian University, Xi' an City, China

- Mingqiang Wei, Nanjing University of Aeronautics and Astronautics, China

- Yücel Yemez, Koç University, Turkey

During 2021, as usual, the journal published a special issue containing the best papers from the Computer Graphics International Conference (CGI'2021) organized by the Computer Graphics Society (CGS). The conference was scheduled to be in Geneva, but due to the pandemic, was finally held online from 6 to 10 September 2021.

This journal could not exist without the strong support and collaboration of many people. We would like to thank several key persons at Springer who are supporting us continuously, our senior editor, Rachel Moriarty, her senior editorial assistant, Annette Hinze, and the project coordinator, Jutta Daum. We are also grateful to Murugeswari Rangasamy for answering and dealing with the daily questions we receive from our authors and reviewers. We would like to thank all of them for 
their continuous great assistance in a journal that handles 12 issues yearly and publishes about 300 papers a year.

Last but not the least, we thank all our authors who have submitted their work to the Visual Computer as well as our readers. We thank them for their interest and support in our journal.

We look forward to 2022 as an exciting New Year for the Visual Computer and we encourage authors to submit the best of their work anytime. In addition, the CGI'2022 call for papers is now open for submission and we hope to get the best papers selected and published in the September CGI special issue of the Visual Computer.
We wish everyone to stay safe and healthy in this difficult pandemic time.

Nadia Magnenat Thalmann

Editor-in-Chief

The Visual Computer

Publisher's Note Springer Nature remains neutral with regard to jurisdictional claims in published maps and institutional affiliations. 